Revue des patrimoines

\title{
Images du cheval à la cour : les programmes iconographiques (Italie-France, XVIe- XVIIe siècles)
}

\section{Elisabetta Deriu}

\section{OpenEdition}

\section{Journals}

Édition électronique

URL : http://journals.openedition.org/insitu/11976

DOI : 10.4000/insitu. 11976

ISSN : 1630-7305

Éditeur

Ministère de la culture

Référence électronique

Elisabetta Deriu, «Images du cheval à la cour : les programmes iconographiques (Italie-France, XVleXVIle siècles) », In Situ [En ligne], 27 | 2015, mis en ligne le 02 novembre 2015, consulté le 19 avril 2019. URL : http://journals.openedition.org/insitu/11976 ; DOI : 10.4000/insitu.11976

Ce document a été généré automatiquement le 19 avril 2019

\section{(c)}

In Situ Revues des patrimoines est mis à disposition selon les termes de la licence Creative Commons Attribution - Pas d'Utilisation Commerciale - Pas de Modification 4.0 International. 


\title{
Images du cheval à la cour : les programmes iconographiques (Italie- France, XVIe-XVIIe siècles)
}

\author{
Elisabetta Deriu
}

Je tiens tout particulièrement à remercier M. Frédéric Henri, aujourd'hui chargé de la gestion et des partenariats au château d'Oiron, non seulement pour l'intérêt qu'il a manifesté pour ma recherche mais aussi pour les renseignements pratiques qu'il a eu la gentillesse de me fournir lors de l'organisation de mon déplacement dans les Deux-Sèvres. Je n'aurais jamais pu effectuer mon reportage au Castello Pandone de Venafro sans l'autorisation du Ministero per i Beni e le Attività Culturali, que le surintendant par interim d'alors (Molise), M. Ruggero Pentrella, m'avait fait parvenir dans les meilleurs délais. Merci également à M. Mario Di Sandro, pour avoir facilité mon travail sur le terrain en m'ouvrant les portes du château en qualité d'opérateur chargé de l'accueil et de la surveillance.

1 Les sources iconographiques spécifiquement consacrées au cheval et témoignant de sa présence au sein des cours italiennes et françaises pendant les $\mathrm{XVI}^{\mathrm{e}}$-XVII ${ }^{\mathrm{e}}$ siècles sont très nombreuses et riches dans leur diversité ${ }^{1}$. On peut isoler quatre groupes de documents particulièrement intéressants, tant pour l'étude des pratiques équestres et des procédés de présentation par l'image du savoir lié au cheval que pour leur valeur documentaire : les dessins des artistes ayant posé sur le cheval un regard de connaisseur; les recueils de gravures ayant pour objet la constitution d'un « haras virtuel »; les cycles de fresques qui ornent certaines résidences princières en France et en Italie; les recueils de patrons, utiles pour la réalisation de plusieurs éléments du harnachement équestre.

2 Les modalités d'élaboration du thème équestre varient évidemment selon les techniques d'exécution employées, le type de support et les souhaits du commanditaire. Parmi les critères qui régissent la mise au point des programmes destinés au milieu curial, trois en particulier exaltent le cheval en tant qu'animal princier :

3 - la sérialité, par la multiplication des prises de vue du cheval dans le but de mieux en appréhender la morphologie, 
4 - la mesure, se manifestant par le respect de la proportion des parties anatomiques du cheval,

5 - l'attention aux exigences curiales, pour le soin avec lequel sont représentés les détails définissant ces équidés comme des montures princières, tout en amplifiant le prestige de la dynastie destinataire du programme.

\section{Les dessins}

6 À la Renaissance, l'étude de l'anatomie et des attitudes du cheval est à l'origine d'une production de dessins particulièrement abondante. L'investigation scientifique n'est pas toujours le but principal de ces analyses minutieuses de la morphologie des équidés. Nombre de ces études, qui ont pourtant une grande valeur technique, ont en effet été conçues et produites pour répondre aux exigences propres du milieu curial. Les dessins de Pisanello, par exemple, dont la qualité du graphisme est exceptionnelle, ne sont généralement pas une fin en eux-mêmes mais servent de base pour l'exécution de médailles, fresques et portraits. Pareillement, les traités manuscrits alliant dessins, textes et calculs que bon nombre d'artistes consacrent aux proportions équestres, servent eux aussi de patrons pour la réalisation de tableaux et de monuments équestres. On ne peut pas exclure pourtant que certains de ces dessins aient pu être exécutés en vue de la constitution d'un ou plusieurs recueils ayant pour thème le cheval, puisque la constitution de séries d'attitudes ou de types chevalins est très en vogue tout au long de la Renaissance.

7 Comme le remarque Michel Pastoureau, « dans le bestiaire pisanellien [...] le cheval est le roi $^{2} »$. Pisanello exécute une quantité considérable de dessins ayant pour sujet des montures, le plus souvent princières. Parce que cet artiste «couvre " des événements curiaux de première importance, on pourrait à juste titre le définir comme le chroniqueur imagier des différentes pratiques équestres en vigueur dans les cours italiennes et étrangères. Il est en effet appelé à prêter son concours lors de grands événements: par exemple, le concile de 1438-1439, ouvert à Ferrare et transféré à Florence, et réunissant les principales maisons princières autour des questions concernant leur rôle en défense de la foi chrétienne ${ }^{3}$. Une grande partie de l'œuvre dessinée pisanellienne est précisément issue de l'observation des montures dans ces circonstances. Tel est le cas de ses séries de têtes de chevaux aux narines fendues ${ }^{4}$ ou de ses études d'arrière-trains, criantes de vérité ${ }^{5}$. Il en est de même pour son célèbre Mulet harnaché foisonnant de détails: l'un des plus précieux parmi eux est sans doute la représentation d'un petit dispositif composé de deux parties semi-sphériques richement historié, et permettant de gérer la longueur les rênes, ou de les bloquer ${ }^{6}$.

On pourrait multiplier les exemples à l'infini, tant ces études paraissent vivantes par leur trait rigoureux et en même temps agréable. Les dessins que Pisanello consacre aux montures princières répondent donc aux trois critères de représentation déjà cités. Et pourtant, ce n'est pas grâce à ces cartons dessinés que Pisanello assure sa réussite dans le milieu curial : ces matériaux sont, au contraire, confidentiels et difficilement accessibles. Par sa nature, chaque dessin est un exemplaire unique, dont on ignore dans quelle mesure il circule en dehors de l'atelier de l'artiste. Pisanello s'en sert de base pour d'autres réalisations, notamment les médailles pour lesquelles il est célèbre : celles-ci sont, au contraire, fabriquées en grand nombre et destinées par leur nature à circuler. 
Pourtant, il est indéniable que ce type de support ne permet pas le même degré d'adhésion à la réalité du sujet équestre traité que les dessins. Or, si le regard de l'historien d'art équestre privilégie ces derniers en leur conférant la valeur de témoignage à cause de leur réalisme,

Il serait [...] anachronique de croire que, dans l'Italie de la première moitié $\mathrm{du}_{\mathrm{Xv}} \mathrm{e}^{\mathrm{e}}$ siècle, l'observation et la représentation réaliste de la nature soient pensées comme des moyens plus nobles ou plus efficaces que d'autres pour accéder à la connaissance et à la vérité 7 .

9 C'est particulièrement frappant dans ce cas, puisque dans les traités d'art équestre postérieurs à l'œuvre de Pisanello, la qualité de la représentation de la figure équestre est nettement inférieure à celle sur laquelle se fonde aujourd'hui le renom des dessins pisanelliens. Parce qu'à la Renaissance, "le vrai c'est le verbe ${ }^{8}$ ", les images, pourtant de plus en plus employées pour éclairer le texte des traités équestres, ne sauraient en aucun cas se substituer à celui-ci. L'alliance entre texte et images n'est pourtant pas une nouveauté introduite par les écrivains d'équitation, cette technique ayant d'abord été employée par les artistes chargés de la représentation du cheval en milieu curial.

Il existe d'autres recueils de dessins de chevaux, ou de parties de l'anatomie équine, donnant une analyse des proportions très poussée, sous forme d'annotations et de calculs de la main de l'artiste. Les exemples les plus connus en sont un modèle chevalin attribué à Andrea del Verrocchio, de la fin du $\mathrm{xV}^{\mathrm{e}}$ siècle ${ }^{9}$, l'étude raphaëlienne d'une des statues équestres anciennes du Quirinal à Rome $1515-1520^{10}$, la série d'études inspirées des travaux de Léonard de Vinci faisant partie du Codex Huygens, et datables des années 1550-1580 ${ }^{11}$. Dans les trois cas, le texte et/ou les calculs sont inscrits à l'intérieur même de la figure chevaline, ou de façon à épouser le profil de celle-ci, dans le but de mieux en établir les proportions. L'unité de base principale de toute mensuration est généralement la testa, ou tête du cheval, selon un système déjà appliqué à l'homme par Vitruve, et pouvant être ultérieurement fractionné. Léonard pousse ce système à l'extrême, réussissant à mesurer les unités les plus petites à partir de trois types de données en particulier: tête, $1 / 16^{e}$ de tête et minuti (litt. «menus»), pouvant être fractionnés ultérieurement si besoin est. Aussi, selon Erwin Panofsky, Léonard serait-il parvenu à calculer des unités d'une valeur d' $1 / 900^{\circ}$ de la longueur du cheval, améliorant les records déjà atteints par L. B. Alberti et Dürer dans le domaine des mensurations humaines, et se situant respectivement à $1 / 600^{\mathrm{e}}$ et $1 / 800^{\mathrm{e} 12}$.

11 Parce qu'ils étayent le paradigme complet du modèle équin, ces calculs savants témoignent de l'effort de définir un idéal du cheval parfait, par l'observation des nombreux et différents types de chevaux existants. Si les équidés sont l'unique objet de ces dessins, ou séries de dessins, l'étude de leurs parties ne saurait être une fin en soi. Les auteurs respectifs de ces dessins sont avant tout des artistes qui sondent la morphologie du cheval dans le but d'en exploiter les particularités lors de la pratique de leur profession : s'approprier le modèle par l'image et le calcul signifie mieux respecter ses proportions naturelles au moment de le reproduire. Parmi les maîtres cités, Léonard de Vinci multiplie tout particulièrement les études dont le but final est la réalisation de sculptures destinées au milieu curial.

12 Les trois critères à partir desquels on peut appréhender la production iconographique ayant pour objet le cheval s'appliquent donc également à ces dessins de maître : la mesure est à la base même de l'exécution des études ; l'établissement de séries de modèles permet d'établir des méthodes de comparaison; et si ces recueils de dessins font la part belle à 
l'investigation scientifique, ils n'en sont pas moins un instrument susceptible d'aider l'artiste à satisfaire les exigences du milieu curial en matière de représentation du thème équestre puisqu'ils multiplient les études comparatives dont le but final est la réalisation de sculptures destinées, précisément, à la cour. Confidentiels, difficilement accessibles par leur nature, l'on ignore dans quelle mesure ces recueils d'études circulent en dehors de l'atelier de l'artiste. Il n'en va pas de même pour les recueils de planches gravées, destinées à être reproduites à de nombreux exemplaires et visant un public bien plus large, mais toujours de qualité.

\section{Les recueils de planches gravées, ou le « haras virtuel »}

Ce type de production artistique éminemment curiale répond à deux impératifs principaux : flatter le dédicataire en lui adressant un " produit » entièrement consacré à l'un des animaux princiers par excellence ; lui fournir une synthèse du savoir disponible sur ce sujet par le recours à l'image et au texte. Il reste au moins deux recueils datant de l'époque de la Renaissance, et ayant inspiré la production de séries gravées de scènes équestres des siècles suivants : l'Equile du flamand Jan van der Straet, dit Stradanus (1523-1605), dessiné par l'artiste sans doute dans les années 1530, gravé par Hendrik Goltzius, Jérôme Wierix et Adriaen Collaert, et édité par Philippe Galle à Anvers à partir des années $1610^{13}$; la série de Chevaux de différents pays dessinés par Antonio Tempesta (1555-1630) et circulant sous forme de planches gravées autour de 1580, par les soins de Giulio Ortino ${ }^{14}$.

Ces recueils se composent de séries de portraits ou de scènes équestres ayant pour décor des paysages évoquant la zone géographique de provenance du cheval représenté. En général, chaque planche comporte le nom du type de cheval pris en considération, ou un court descriptif de la scène ou de l'attitude reproduite; ensuite, une légende en latin insérée au-dessous du portrait, ou de la scène, fournit des informations souvent savantes, en particulier les qualités physiques et morales des équidés dont on illustre l'attitude. L'examen parallèle des deux recueils permet d'en appréhender les nombreuses ressemblances : hormis les différences stylistiques, les types chevalins représentés sont les mêmes dans les deux cas, et le texte latin des légendes illustrant l'Equile de Stradanus est identique à celui qui commente les séries de Tempesta. L'état actuel des connaissances ne permet pas d'établir avec certitude lequel de ces deux artistes a constitué le premier ces séries de tableaux équestres, d'autant que Tempesta se serait formé à l'école de Stradanus ${ }^{15}$. Quel que soit l'auteur du concept de la série de planches équestres, celui-ci a pour but la constitution d'un haras virtuel ayant un double intérêt: inventorier les connaissances disponibles sur chaque type de cheval prisé à la Renaissance ; les présenter de façon à pouvoir offrir à un personnage princier tous les chevaux du monde, pour ainsi dire, dont les qualités ressortent grâce à l'image claire et soignée et grâce au texte explicatif. D'ailleurs, les titres mêmes des recueils cités renvoient à cette visée d'exhaustivité : l'ouvrage de Stradanus, en particulier, prend le nom d'equile, c'est-à-dire haras et comporte quarante-deux planches représentant autant de types ou attitudes chevalines; l'intitulé de celui de Tempesta, en revanche, met davantage l'accent sur la provenance géographique des chevaux, les planches étant dans ce cas une trentaine. L'effort de systématisation des connaissances qui caractérise ces deux recueils les rapproche des traités d'art équestre dont la plupart fournissent des descriptifs détaillés 
des différents types de chevaux, répartis notamment selon des critères géographiques. Tant l'Equile que les Chevaux de différents pays peuvent se prévaloir de dédicataires illustres ayant la réputation de connaisseurs : l'Equile est, en effet, aussi connu sous le titre de Haras de Jean d'Autriche, ce personnage ayant été le patron de Stradanus jusqu'en 1578 ; le recueil de Tempesta porte une dédicace de l'éditeur au jeune Virginio Orsini ${ }^{16}$. Parmi les critères susceptibles de définir l'iconographie ayant pour thème le cheval, la notion de sérialité est donc celle qui permet de mieux appréhender la production de planches gravées. Sa double portée est dans ce cas particulièrement évidente : la série d'attitudes et de types chevalins possède une valeur didactique et sert aussi de prétexte à la répétition du motif princier qu'est le cheval, véhiculant des valeurs propres au milieu curial.

Le thème de l'iconographie équestre en milieu curial peut également être abordé par l'étude des fresques ayant pour thème le cheval, et dont la finalité principale est la célébration de l'environnement aristocratique destiné à les abriter. Certaines d'entre elles ont un but explicitement documentaire qui permet à l'historien de les exploiter en tant que témoignage de la diffusion de certaines pratiques équestres.

\section{Les grands cycles de fresques équestres}

Les grandes scènes que cette technique permet de représenter peuvent orner soit les lieux consacrés à la stabulation des chevaux et à l'entretien de l'équipement, soit les pièces se trouvant à l'intérieur même de la résidence princière. En Italie, se multiplient les cycles de fresques pour décorer l'intérieur des palazzi nobiliaires et ayant pour sujet le cheval et les pratiques équestres. Pour n'en citer que quelques-uns, le palais Schifanoia à Ferrare abrite la représentation d'une course de palio, due au pinceau de Francesco del Cossa et datant de 1467-1470 ${ }^{17}$; le palais ducal de Mantoue compte un grand cycle représentant un combat des cavaliers exécuté par Pisanello, vraisemblablement entre 1425 et $1455^{18}$. Dans tous les cas, la fresque orne de vastes pièces, au cœur même de la maison du prince, le plus souvent une salle de réception: c'est le motif équestre qui accueille le visiteur de qualité, tout en témoignant du rang de l'hôte.

En France, il existe aussi plusieurs exemples de fresques, mais elles ornent, en revanche, les lieux consacrés à la population équine de la maison princière. L'intérieur des écuries du château d'Escars, près de Limoges, reproduit des cavaliers maniant leurs montures, datables des années 1515-1520, et qui « séduisent par la justesse des attitudes saisies par l'artiste et une forte impression de mouvement ${ }^{19}$ ». Le château de Villeneuve-Lembron (Puy-de-Dôme) présente une scène de combat mythologique elle aussi très vivante, et qui date probablement des années 1590 . On compte également des témoignages d'époque plus tardive et aux dimensions plus réduites, comme le petit cheval qu'abrite le château de Creuilly en Normandie, prouvant que la vogue de la fresque équestre se maintient au moins jusqu'au début du XVII ${ }^{\mathrm{e}}$ siècle $^{20}$.

$\mathrm{Au}$ sein de cette production, le goût se développe, tant en Italie qu'en France, pour la représentation grandeur nature de chevaux bien identifiables et issus de l'élevage ou appartenant à l'écurie de la maison princière qui souhaite en perpétuer ainsi le souvenir. La mode du portrait équestre mural parait avoir été inspirée tout particulièrement par le programme réalisé par Giulio Romano au palais du Tè de Mantoue pour les Gonzague au cours des années 1520-1530. Le château d'Oiron dans les Deux-Sèvres garde des traces de 
l'adoption par la France de cette mode du grand portrait équestre : datant de 1540, il se rapproche donc de l'époque de la fin des travaux au palais du Tè, en 1534. Cela permet de supposer que la salle des Chevaux mantouane aurait inspiré la réalisation des portraits ayant jadis décoré les murs de la galerie basse de la demeure des Gouffier à Oiron.

\section{Château d'Oiron (Deux-Sèvres)}

La Galerie basse ou galerie des Chevaux, à gauche dans la cour, a été édifiée par Artus Gouffier entre 1515 et $1519^{21}$.

Figure 1

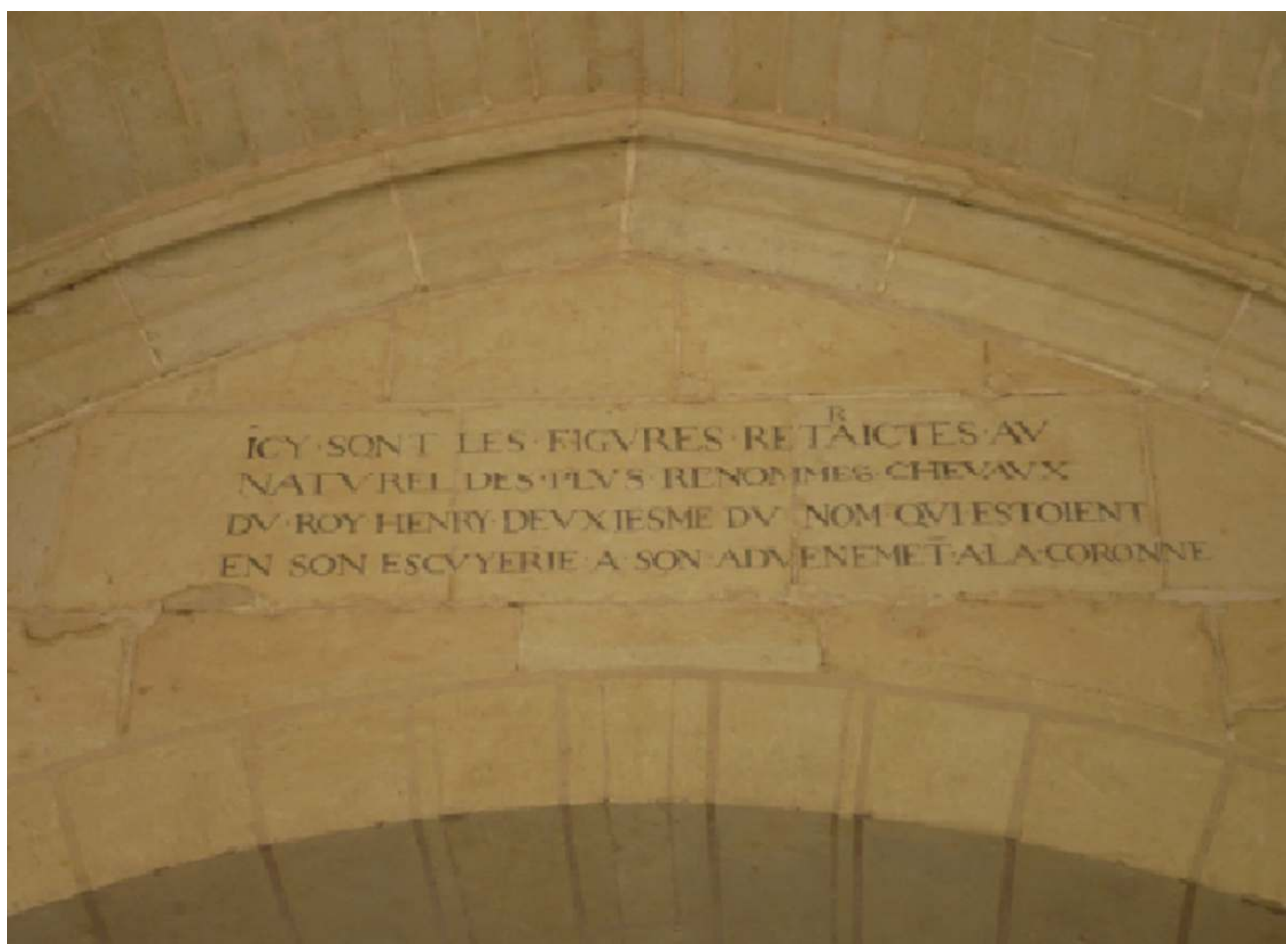

Château d'Oiron (Deux-Sèvres) : galerie basse.

Phot. Deriu, Elisabetta, 2008. (c) Elisabetta Deriu.

Son fils, Claude Gouffier, grand écuyer d'Henri II, y fait représenter les marques des chevaux appartenant au roi (fig. 1). Au nombre de soixante-dix et peintes au pigment noir, elles renvoient soit à des haras pour la plupart italiens, soit aux races de François $\mathrm{I}^{\mathrm{er}}$, Henri II et Charles IX. Cette galerie avait également abrité les portraits « au naturel » des chevaux intégrant les écuries royales à l'époque de l'avènement d'Henri II, ainsi qu'en témoigne l'inscription située au fond de la galerie ${ }^{22}$. En 1875, Léon Palustre inventorie toutes les marques, dont il fournit également une transcription. Les marques royales, non publiées, font en revanche l'objet d'une description détaillée ${ }^{23}$. Quarante sur les soixantedix marques que comporte la galerie des Chevaux d'Oiron sont accompagnées d'une légende qui en détaille l'origine (fig. 2). Ayant de toute évidence fait l'objet d'une restauration récente, la plupart d'entre elles sont clairement lisibles, tout comme leur légende. Toutefois, en effectuant sur le terrain une comparaison entre la totalité des marques existantes et le corpus présenté par Léon Palustre, nous avons relevé un certain nombre de discordances - en particulier pour ce qui est de la morphologie des marques, 
et du texte les illustrant - et plusieurs omissions : marques dont la morphologie présente des différences par rapport à la transcription proposée par Léon Palustre, marques dont la légende ne coïncide pas avec son interprétation ${ }^{24}$.

Figure 2

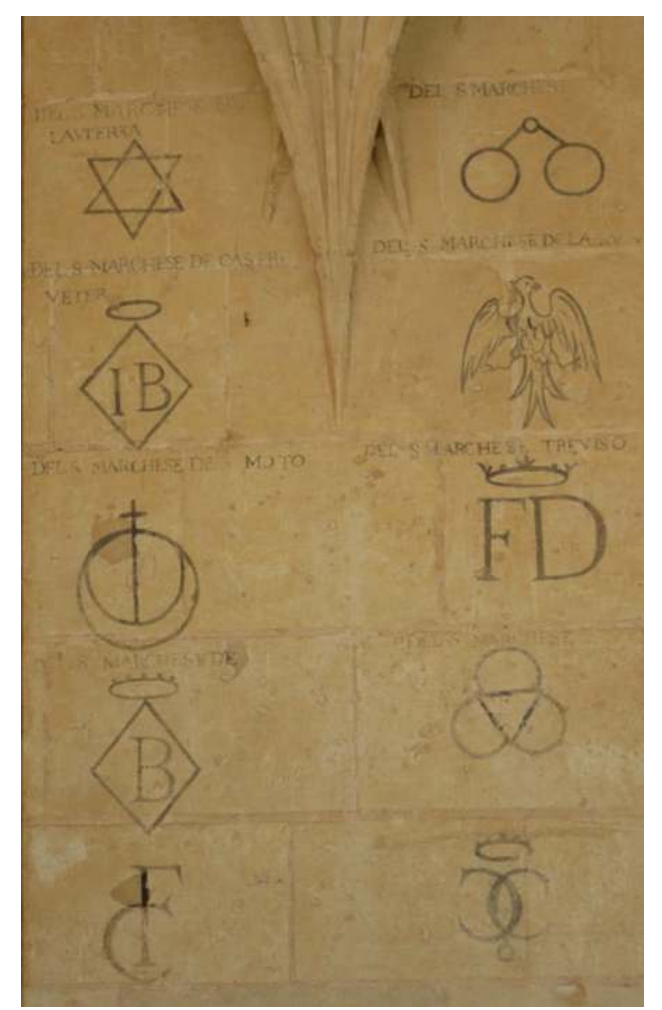

Château d'Oiron (Deux-Sèvres) : les marques équestres.

Phot. Deriu, Elisabetta, 2008. (c) Elisabetta Deriu.

21 En se penchant sur les influences italianisantes, Pascal Liévaux avance l'hypothèse que d'autres exemples de fresques équestres situés dans la Péninsule seraient encore à découvrir ${ }^{25}$. Effectivement, il existe un précédent beaucoup moins connu que le palais du Te et montrant que le cycle mantouan est loin d'être l'archétype du genre : il s'agit d'une série de dix-neuf portraits se trouvant dans la salle de réception du Castello Pandone de Venafro, près d'Isernia (Molise) et dont l'exécution remonte aux années 1522-1524.

\section{Castello Pandone, Venafro (Isernia), Molise}

Fondé par les Lombards autour du $\mathrm{x}^{\mathrm{e}}$ siècle, faisant l'objet des assauts des Normands au cours du XII ${ }^{\mathrm{e}}$, agrandi par les Angevins au XIII ${ }^{\mathrm{e}}$ siècle puis détruit par un incendie au XIV ${ }^{\mathrm{e}}$ siècle, ce castello passe aux mains des Aragon dans les années 1440. C'est alors que s'y établit Francesco Pandone, fraîchement nommé comte. La famille Pandone y réside jusqu'à la fin des années 1520 . 


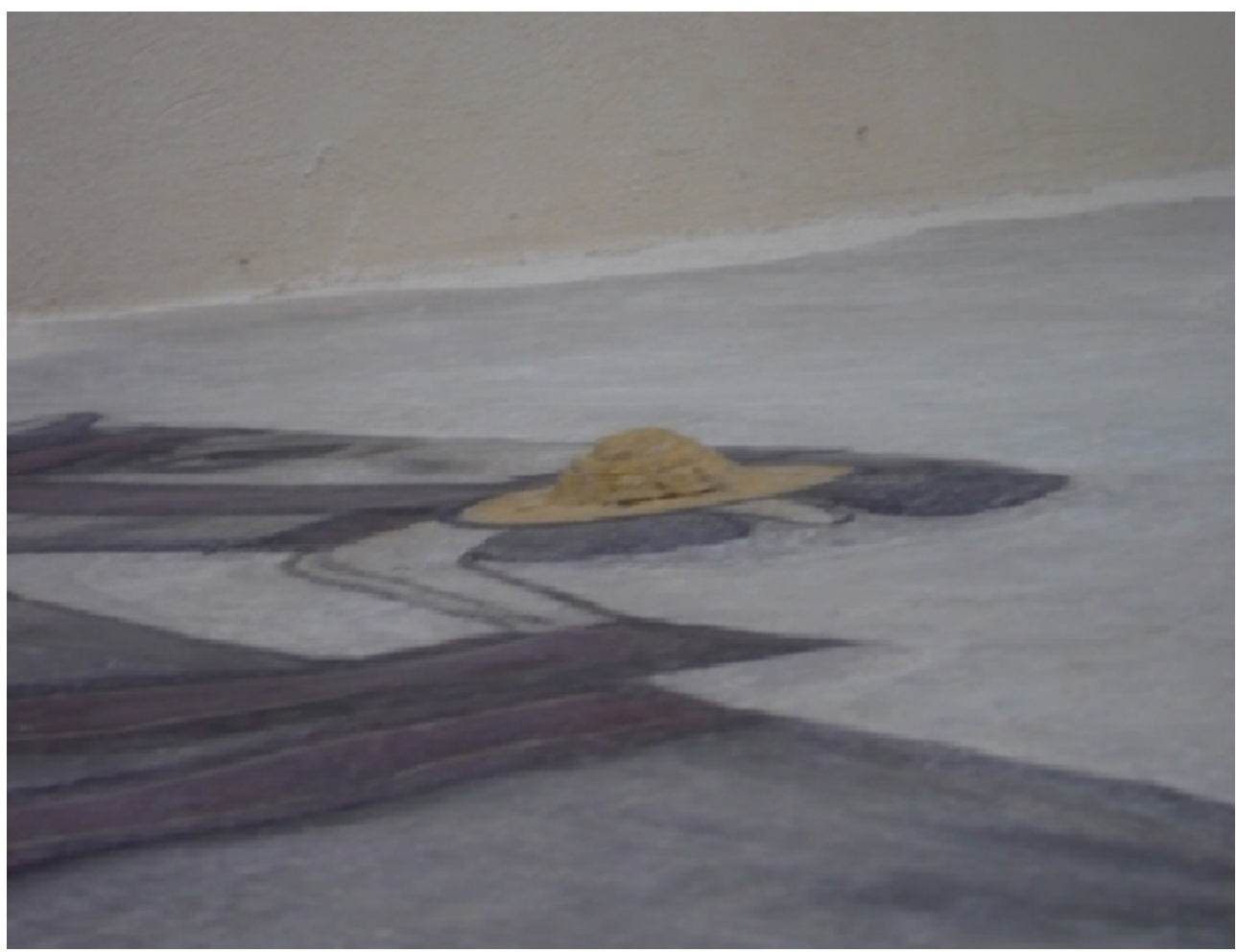

Castello Pandone, Venafro (Italie). La fresque en bas-relief méplat (« stiacciato »).

Phot. Deriu, Elisabetta, 2008. (c) Elisabetta Deriu.

L'entrée et les salles de réception sont ornées de vingt et un portraits de chevaux issus du haras des seigneurs de Pandone et exécutés par des artistes anonymes en combinant deux techniques : la fresque et le bas-relief méplat, c'est-à-dire suggérant le modelé des formes, mais présentant une diminution de l'épaisseur des premiers plans (fig. 3). Datant des années 1524-1527, ces fresques furent partiellement détruites au début du XVIII siècle, lors d'importants travaux de modification et de rénovation. Il reste des dessins préparatoires étonnamment bien conservés des portraits dont on a détruit l'enduit portant la fresque (fig. 4) : il s'agit des deux chevaux ornant l'entrée de la résidence, située dans la cour interne, et de neuf autres montures parmi celles ayant été peintes dans les salles de réception. 
Figure 4

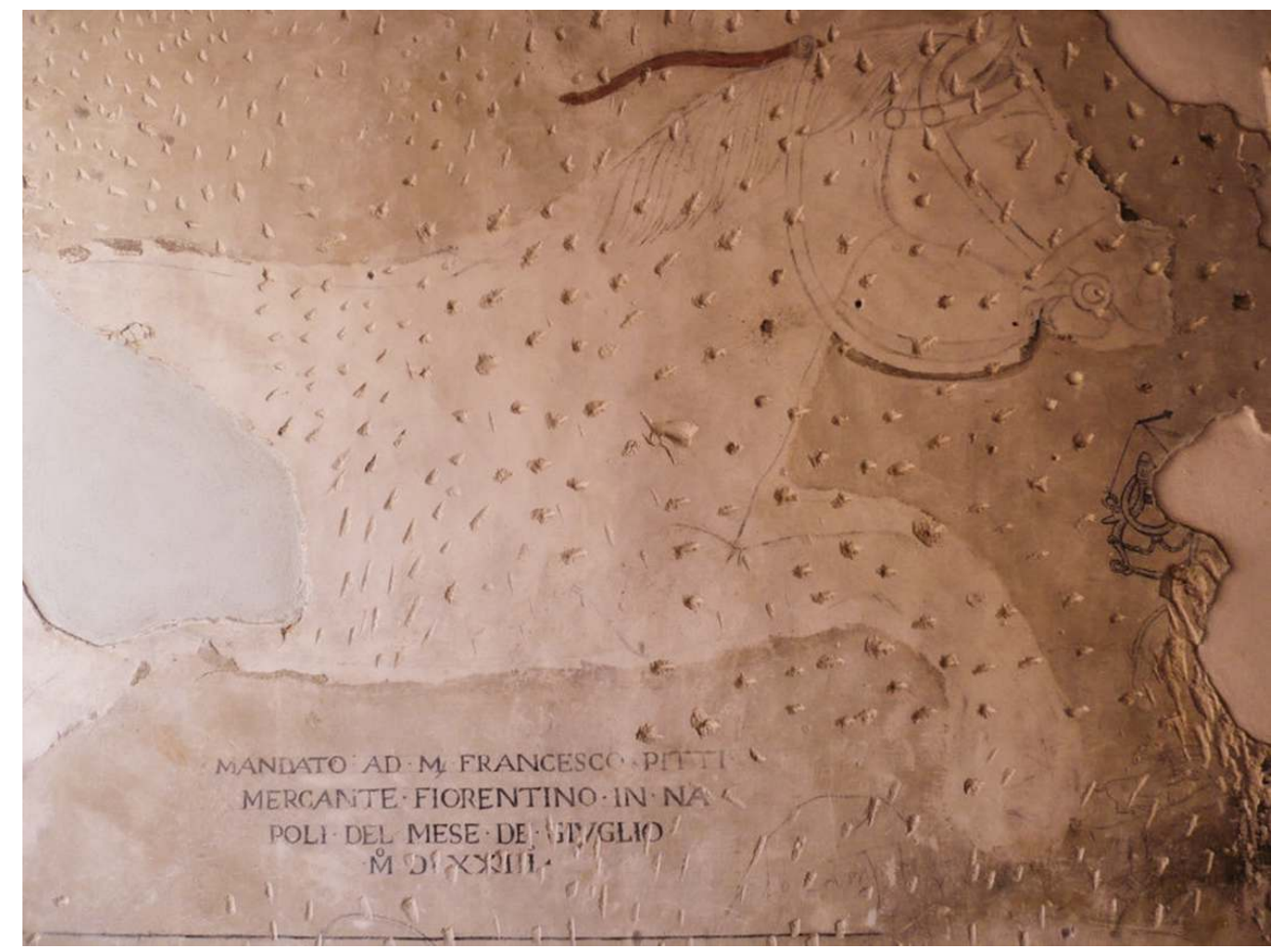

Castello Pandone, Venafro (Italie). Les fresques : dessin préparatoire.

Phot. Deriu, Elisabetta, 2008. (c) Elisabetta Deriu.

Chaque portrait a été exécuté en suivant un modèle bien précis, quasiment une fiche établie selon des critères qui rappellent de près ceux régissant la rédaction d'un registre d'écurie (fig. 5). 


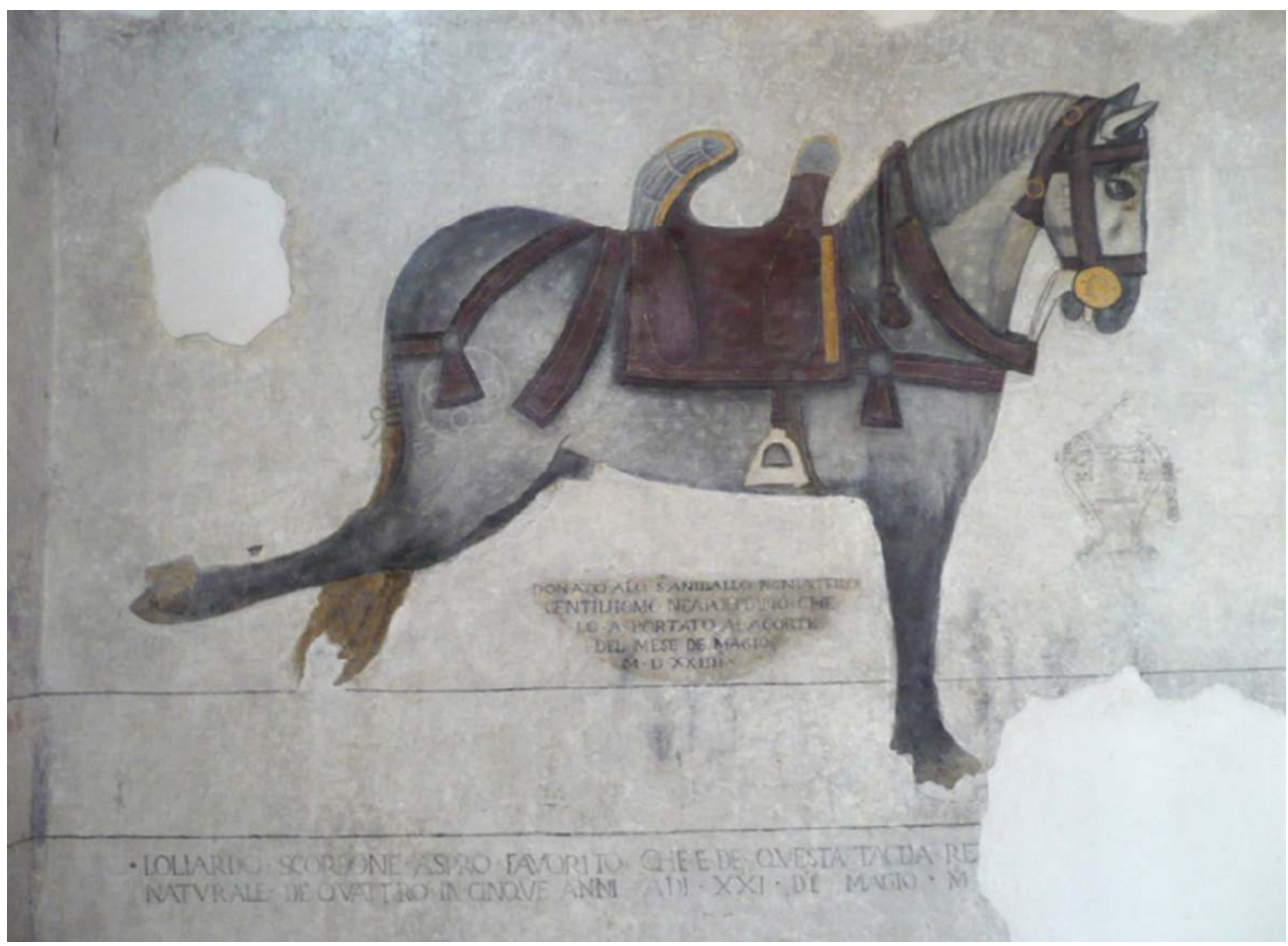

Castello Pandone, Venafro (Italie). Profil de la monture.

Phot. Deriu, Elisabetta, 2008. (c) Elisabetta Deriu.

Contrairement aux registres émanant des établissements équestres princiers, ce cycle de fresques équestres présente donc la particularité de définir la taille des chevaux représentés. Si les légendes n'indiquent pas les dimensions, le portrait en lui-même, ayant été exécuté d'après nature, montre que la hauteur au garrot de ces montures oscille plus ou moins entre 1,50 et 1,70 m. Les chevaux issus des races de la maison Pandone sont des montures réellement princières, à la fois par la beauté de leur modèle, de leur robe, par la richesse du harnachement, tout autant que par la marque prestigieuse qu'elles portent. Leur pose est statique, à trois exceptions près : les deux chevaux de l'entrée, représentés au galop, le cheval gris pommelé exécutant une ruade et dont l'attitude dédramatise la solennité émanant des autres montures : s'agit-il d'un virtuose des airs, ou d'une monture au sale caractère, se nommant, nomen omen, "Scorbone aspro ": "cheval âpre et hargneux » (fig. 6) ? Un inventaire exhaustif des portraits du castello Pandone, effectué dans les années 1980, est donc déjà disponible et exploitable en vue d'approfondissements ultérieurs ${ }^{26}$. 


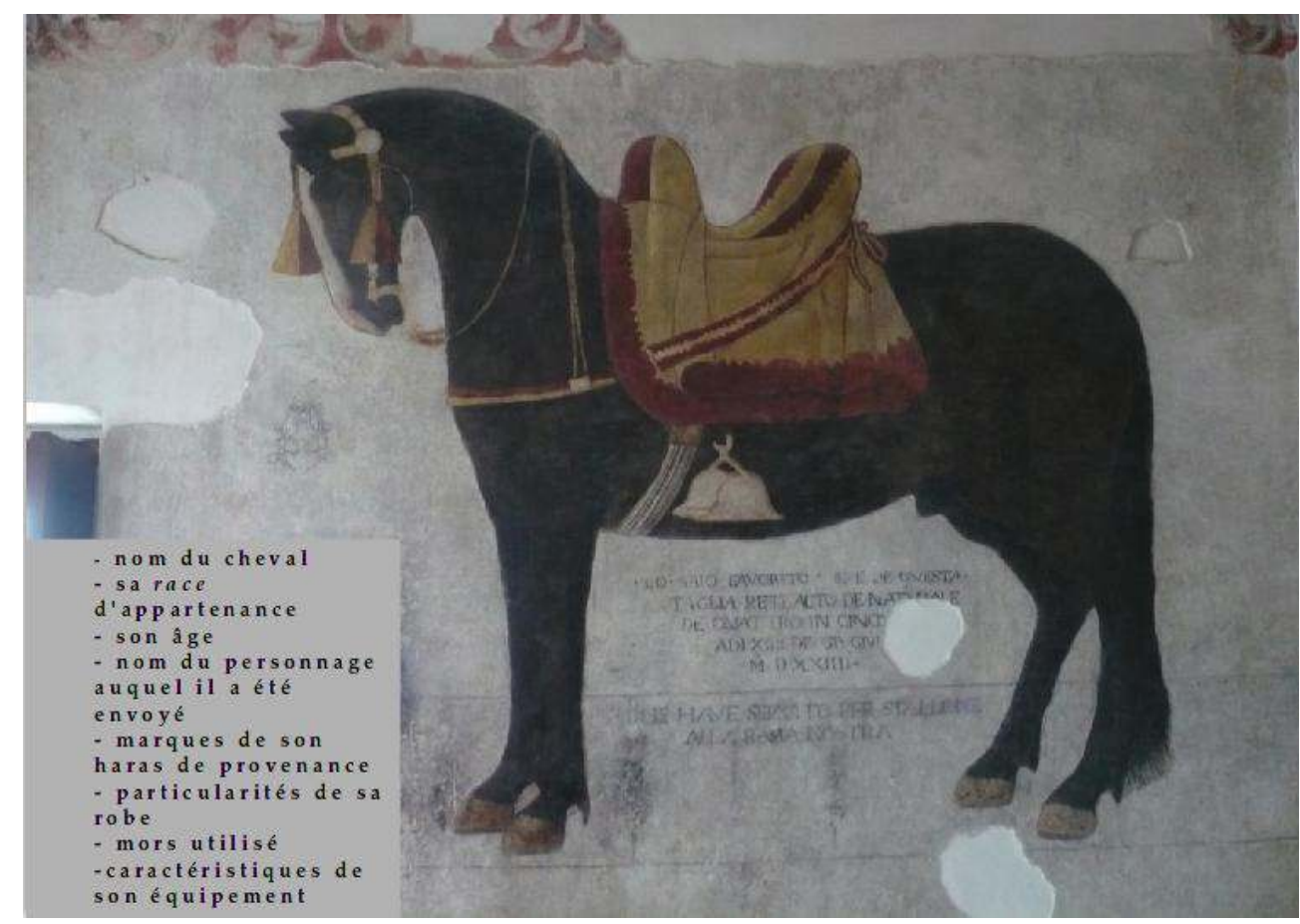

Castello Pandone, Venafro (Italie). Les fresques : « Scorbone Aspro ».

Phot. Deriu, Elisabetta, 2008. (c) Elisabetta Deriu.

Les exemples cités fournissent amplement matière à réflexion concernant le choix du sujet, les modalités d'exécution et la finalité de ces grands cycles de portraits équestres. Si les œuvres de Mantoue, Oiron et Venafro possèdent des caractéristiques originales, il est possible d'en appréhender certaines constantes qui permettent de mieux les définir en tant que produits propres aux milieux curiaux italiens et français. D'abord, le choix du sujet: dans les trois cas, il s'agit de chevaux non montés, peints en couleur, grandeur nature, ou légèrement plus grands que nature. Ensuite, la technique d'exécution: si l'artiste des Gouffier à Oiron opte pour la peinture sur cuir, ceux de Pandone et de Gonzague privilégient la fresque. En ce qui concerne les finalités, ces cycles se trouvent toujours au cœur de la résidence princière, et concourent au prestige de la maison d'une manière double : elles ont d'abord été conçues comme témoignage, et possèdent donc une valeur documentaire aux yeux des commanditaires eux-mêmes; en même temps, la répétition du motif équestre et son amplification par le choix de l'emplacement et par la représentation en grandes dimensions confèrent à ces cycles le statut d'une merveille destinée à impressionner favorablement les visiteurs de prestige, tout en rendant hommage au patron des lieux.

27 Le thème de l'iconographie équestre en milieu curial peut également être abordé par l'analyse d'un autre type d'objets?/de support e matériaux où l'image revêt un rôle fondamental, mais qui ne sont pas spécialement consacrés au cheval: les livres de patrons, où les notions de « série » et de «mesure » triomphent et ont alors une valeur éminemment pratique. Parce qu'ils contiennent des renseignements utiles pour l'étude de la tenue du cavalier et du harnachement du cheval, ces documents sont toujours $\mathrm{du}$ plus grand intérêt pour l'historien de l'art équestre se penchant sur les consommations en milieu curial. 


\section{Les « libri del fare » : recueils de patrons, et registres de la garde-robe}

Silvio Leydi recourt à l'expression "libro del fare», fare signifiant "faire, réaliser, confectionner ", pour évoquer les compétences artisanales qui sont à l'origine du Libro del Sarto du tailleur milanais Gian Giacomo del Conte, actif au milieu du XVI ${ }^{\mathrm{e}}$ siècle ${ }^{27}$. Mélange de dessins, de patrons en papier, d'estampes et de notes autographes du tailleur, ce volume provient de l'atelier de couture que celui-ci tenait à Milan. Par leurs caractéristiques, d'autres sources peuvent également être définies comme "libri del fare ». C'est le cas, notamment, des nombreux inventaires et des Quadernacci (que l'on peut rendre par " cahiers de brouillon ») provenant des archives de la garde-robe des Médicis à Florence, un organisme curial spécialement consacré à la gestion des biens de la Maison. À l'instar du livre de patrons milanais, ces dossiers florentins se caractérisent par l'hétérogénéité des matériaux qui les composent: textes, mensurations, esquisses, patrons. Par leur nature, les livres de patrons se prêtent à une double exploitation des données abondantes et diversifiées qu'ils comportent: d'abord, ils témoignent des modalités de consommation en vigueur dans les milieux qui les produisent; en même temps, ils renseignent sur le savoir-faire nécessaire pour la réalisation de nombreux objets relevant des pratiques équestres. Ces recueils livrent donc de précieux indices sur l'évolution des modes équestres en milieu curial et sur la façon dont les professionnels répondent aux exigences des courtisans. Ainsi, le Libro del Sarto fournit des indications précises sur les modes venant de l'étranger : en témoigne la tenue conçue pour une dame souhaitant se produire à cheval habillée à l'orientale, et maniant «à la genette ». De même, le tailleur milanais consigne dans son journal les renseignements utiles pour satisfaire les exigences de certaines catégories de clients : par exemple, la description illustrée de la tenue portée par la suite des hommes d'armes de Ferrante de Gonzague ${ }^{28}$. La particularité de ce précieux livre de tailleur réside dans l'insertion de patrons utiles pour la confection de plusieurs éléments du harnachement équestre. La planche c. 26, par exemple, fournit toutes les données nécessaires "pour faire un vêtement pour un cheval de condition conformément audit dessin", lequel représente un cheval portant une couverture de deuil. Les notes qui accompagnent les patrons précisent également la quantité de drap nécessaire pour la réalisation d'un tel appareil, « compte tenu du tour de l'encolure et de la poitrine »: cette ample couverture ne demande pas moins de huit aunes de drap noir et deux aunes de toile ${ }^{29}$.

Les inventaires et les Quadernacci de la garde-robe florentine constituent une véritable aubaine pour le chercheur, à l'instar des autres matériaux que comportent ces archives : tout historien peut y trouver son compte, quelle que soit la spécialisation qui est la sienne. Les Quadernacci et les inventaires appartiennent en effet à un ensemble de documents vaste et structuré, enregistrant les consommations curiales en fonction des catégories d'objets et de leur destination. Aussi, chaque service de la garde-robe possèdet-il ses propres journaux, registres et catalogues: par conséquent, les Quadernacci et les inventaires contenant les informations relatives aux objets équestres relèvent de l'administration des Écuries médicéennes à Florence. Tout comme le Libro del Sarto, ces registres comportent un nombre important de dessins et de notes éclairant les modalités de fabrication ou même de réparation des accessoires dessinés. Tout ce qui relève de la fabrication et de l'entretien des carrosses est traité séparément, et est à l'origine d'une 
production spécifique : un cahier datant du $\mathrm{XVI}^{\mathrm{e}}$ siècle, par exemple, présente les dessins dont certains sont coloriés - minutieusement exécutés de toutes les pièces composant une voiture ${ }^{30}$.

Qu'ils proviennent de l'atelier d'un tailleur milanais ou d'une structure plus complexe comme la garde-robe des Médicis, les libri del fare sont d'autant plus précieux qu'ils permettent de situer les différents objets et accessoires dans leurs processus de fabrication respectifs, et permettent ainsi à l'historien d'appréhender concrètement les modes curiales.

\section{NOTES}

1. - Elisabetta Deriu est docteure en histoire moderne de l'université Paris Est-Créteil laboratoire de rattachement : Centre pour la Recherche en histoire européenne comparée (2008). Elle a été research fellow au sein de la National Sporting Library and Museum de Middleburg-Virginia (2009), puis visiting researcher à l'université de Dublin (2011). Ayant obtenu sa qualification aux fonctions de maître de conférences en 2009, et de registered teacher (Teaching Council irlandais) en 2011, elle travaille actuellement en tant que professeure d'histoire véhiculée en français dans deux lycées du Convitto Nazionale «Vittorio Emanuele II » de Rome. En parallèle, elle mène des recherches dans les Archives secrètes et à la bibliothèque du Vatican. Voir le site : http:// www.stmoderna.it/deriu-elisabetta_a2908.

2. - PASTOUREAU, Michel. «Un peintre italien en son temps: nordique, héraldique, mélancolique ». Dans Pisanello. Le peintre aux sept vertus. Paris: Réunion des Musées Nationaux, 1996, p. 23.

3. - CORDELLIER, Dominique. "Chronologie ». Dans Pisanello. Le peintre aux sept vertus. Paris : Réunion des Musées Nationaux, 1996, p. 27.

4. - Voir sur la base Joconde: http://www.culture.gouv.fr/Wave/image/joconde/0285/ m503501_d0003140-000_p.jpg ; http://www.culture.gouv.fr/Wave/image/joconde/0285/ m503501_d0003150-000_p.jpg.

5. - Voir sur la base Joconde: http://www.culture.gouv.fr/Wave/image/joconde/0284/ m503501_d0003247-000_p.jpg.

6. - Voir dans la base Joconde: http://www.culture.gouv.fr/Wave/image/joconde/0285/ m503501_d0003170-000_p.jpg.

7. - PASTOUREAU, Michel. «Un peintre italien en son temps: nordique, héraldique, mélancolique ». Dans Pisanello. Le peintre aux sept vertus. Paris : Réunion des Musées Nationaux, 1996, p. 22.

8. - PASTOUREAU, Michel, op. cit., p. 22.

9. - LIEDTKE, Walter. The Royal Horse and Rider. Painting, sculpture and Horsemanship 1500-1800. New York : Abaris Books, 1989, p. 165.

10. - LIEDTKE, Walter, op. cit., p. 111.

11. - PANOFSKY, Erwin. Le codex Huygens et la théorie de l'art de Léonard de Vinci ("The Codex Huygens and Leonardo da Vinci's Art Theory. The Pierpont Morgan Library Codex M.A. 1139“. London, The Warburg Institute, 1940, traduit de l'anglais et présenté par Daniel Arasse). Paris : Flammarion, 1996.

12. - PANOFSKY, Erwin, op.cit., p. 38-39. 
13. - BARONI VANNUCCI, Alessandra Baroni. Jan van der Straet detto Giovanni Stradano: flandrus pictor et inventor. Roma-Milano : Jandi Sapi, 1997, p. 366 ; STRAUSS, Walter L. The Illustrated Bartsch . New York: Abaris Books, 1982, t. 3 (Commentary), p.312-326; sur la genèse des travaux de Stradanus, voir également MARGOLIN, Jean-Claude. "Inventer et découvrir à la Renaissance ». Dans Inventions et découvertes au temps de la Renaissance. Paris : Klincksieck, 1994, p. 128-130. Pour un regard plus récent, voir BARONI, Alessandra, SELLINK, Manfred. Stradanus, 1523-1605: court artist of the Medici. Turnhout: Brepols, 2012, p. 238-289 notamment. Voir le site: http:// www.wittert.ulg.ac.be/fr/flori/opera/vanderstraet/vanderstraet_haras.html\#menu.

14. - BUFFA, Sebastien. The Illustrated Bartsch. New York: Abaris Books, 1983, t. 36, p. 185-212. Voir le site : http://www.harvardartmuseums.org/art/236424.

15. - BENEZIT, É. Dictionnaire critique et documentaire des peintres, sculpteurs, dessinateurs et Graveurs [...]. Librairie Gründ, 1966, t. VIII.

16. - BUFFA, Sebastien, op. cit., t. 36, p. 184.

17. - Voir ANCONA, Paolo d'. Les mois de Schifanoia à Ferrara, avec une note critique sur la restauration récente par Cesare Guidi. Milano : Edizioni del Milione, [1954]. Voir le site : http:// www.wga.hu/cgibin/highlight.cgifile=html/c/cossa/

schifano/2april/2april_8.html\&find=schifanoia.

18. - PACCAGNINI, Giovanni. Il Palazzo Ducale di Mantova. Torino : Eri, 1969, p. 28-29.

19. - Ibid., p. 28-29.

20. - LIÉVAUX, Pascal. Les Écuries des châteaux français. Paris : Monum, Éditions du patrimoine, 2005, p. $49,52$.

21. - Voir sur la base Joconde: http://www.culture.gouv.fr/Wave/image/joconde/0685/ m074302_dangy-3140_p.jpg.

22. - Voir BÉLIME, Magali, MARTIN, Jean-Hubert, MESLET, Jean-Luc. Le Château d'Oiron. Paris : Éditions du Patrimoine, 2000 ; Les trésors du Grand Écuyer, Claude Gouffier, collectionneur et mécène à la Renaissance. Paris : Éditions de la Réunion des Musées Nationaux, 1994.

23. - PALUSTRE, Léon. La Renaissance en France. Paris : A. Quantin Imprimeur-Éditeur, 1875, t. III, p. 235-242.

24. - DERIU, Elisabetta. Le Cheval et la Cour. Pratiques équestres et milieux curiaux, Italie et France (milieu du XVe-milieu du XVIIe siècle). Thèse d'Histoire. Créteil : Université de Paris Est-Créteil Val de Marne, 2008, t. III, p. IV-XLIII.

25. - Sans pourtant mentionner Venafro... LIÉVAUX, Pascal. Les Écuries des châteaux français. Paris : Monum, Éditions du patrimoine, 2005, p. 51.

26. - MORRA, Gennaro, VALENTE, Franco. Il Castello di Venafro. Storia, arte, architettura. Ferrazzano (CB) : Edizioni Enne, 2000 (1ère éd. 1993). Voir également DERIU, Elisabetta. Le Cheval et la Cour. Pratiques équestres et milieux curiaux, Italie et France (milieu du XVe-milieu du XVII ${ }^{\mathrm{e}}$ siècle). Thèse d'Histoire. Créteil : Université de Paris Est-Créteil Val de Marne, 2008, t. III, p. XLIV-LXXI.

27. - LEYDI, Silvio. "Il Libro del Sarto, Milano XVI secolo ». Dans Giovanni Battista Moroni. Il Cavaliere in nero. L'immagine del gentiluomo nel Cinquecento. Milano : Skira editore, 2005, p. 102.

28. - À la Renaissance, les professionnels de l'art équestre et des armes ne portent pas d'uniforme. Ils peuvent néanmoins porter les couleurs de la maison princière qui les emploie.

29. - SCHIAVON, Alessandra. «Parole e cifre : le annotazioni nel Libro del sarto». Dans llLibro del Sarto della Fondazione Querini Stampalia di Venezia. Modena : Edizioni Panini, p. 68.

30. - VACCARI, Maria Grazia. La Guardaroba Medicea dell'Archivio di Stato di Firenze. Edizioni Regione Toscana, 1997, p. 11. 


\section{RÉSUMÉS}

Les sources iconographiques spécifiquement consacrées au cheval et témoignant de sa présence au sein des cours italiennes et françaises pendant les XVIe-XVIIe siècles sont très nombreuses et riches dans leur diversité. Notre contribution examine quatre groupes de documents particulièrement intéressants pour l'étude du cheval et du savoir-faire équestre à la cour : dessins, séries de gravures ayant pour objet la constitution d'un «haras virtuel », cycles de fresques, recueils de patrons.

Courtly images of the horse: iconographical programmes in Italy and France during the sixteenth and seventeenth centuries. The equestrian iconography associated with the French and Italian courts during the sixteenth and seventeenth centuries is rich and diverse. This contribution focuses on four types of documents which are particularly relevant for studying horses and equestrian savoir faire at court: drawings, collections of engravings constituting a 'virtual' stud farm, frescoes cycles and collections of saddlery and sewing patterns.

\section{INDEX}

Mots-clés : cours princières, Italie-France, Oiron (France), Florence (Italie), Mantoue (Italie), Venafro (Italie), XVIe-XVIIe siècles, dessins, gravures, patrons, fresques, marques équestres, Gonzaga, Gouffier, Pandone, Pisanello

Keywords : princely courts, Italy, Florence (Italy), Mantua (Italy), Venafro (Italy), sixteenthseventeenth centuries, engravings, patterns, frescoes, horse brands

\section{AUTEUR}

\section{ELISABETTA DERIU}

docteure en histoire moderne, Université de Paris-Est-Créteil (CRHEC)

elisabettaderiu@hotmail.com 